\title{
Effectiveness of trauma centre verification: a systematic review and meta-analysis
}

\author{
Brice Batomen, MSc \\ Lynne Moore, PhD \\ Mabel Carabali, MD, MSc \\ Pier-Alexandre Tardif, MSc \\ Howard Champion, MD \\ Arijit Nandi, PhD
}

Accepted Feb. 19, 2020

\section{Correspondence to:}

\section{B. Batomen}

Department of Epidemiology

Biostatistics, and Occupational Health McGill University

Meredith Charles House, Room B9

1130 Pine Ave W

Montréal QC H3A 1 A3

brice.batomenkuimi@mail.mcgill.ca

DOI: $10.1503 /$ cjs.016219

\begin{abstract}
Background: There is a growing trend toward verification of trauma centres, but its impact remains unclear. This systematic review aimed to synthesize available evidence on the effectiveness of trauma centre verification.

Methods: We conducted a systematic search of the CINAHL, Embase, HealthStar, MEDLINE and ProQuest databases, as well as the websites of key injury organizations for grey literature, from inception to June 2019, without language restrictions. Our population consisted of injured patients treated at trauma centres. The intervention was trauma centre verification. Comparison groups comprised nonverified trauma centres, or the same centre before it was first verified or re-verified. The primary outcome was in-hospital mortality; secondary outcomes included adverse events, resource use and processes of care. We computed pooled summary estimates using random-effects meta-analysis.
\end{abstract}

Results: Of 5125 citations identified, 29, all conducted in the United States, satisfied our inclusion criteria. Mortality was the most frequently investigated outcome $(n=$ $20)$, followed by processes of care $(n=12)$, resource use $(n=12)$ and adverse events $(n=7)$. The risk of bias was serious to critical in 22 studies. We observed an imprecise association between verification and decreased mortality (relative risk $0.74,95 \%$ confidence interval 0.52 to 1.06 ) in severely injured patients.

Conclusion: Our review showed mixed and inconsistent associations between verification and processes of care or patient outcomes. The validity of the published literature is limited by the lack of robust controls, as well as any evidence from outside the US, which precludes extrapolation to other health care jurisdictions. Quasiexperimental studies are needed to assess the impact of trauma centre verification.

\section{Systematic reviews registration: PROSPERO no. CRD42018107083}

Contexte : Le processus d'audit des centres de traumatologie gagne en popularité, mais ses effets concrets ne sont pas bien connus. La présente revue systématique a cherché à résumer les données probantes disponibles sur l'efficacité de l'audit des centres de traumatologie.

Méthodes : Nous avons effectué des recherches systématiques dans les bases de données CINAHL, Embase, HealthSTAR, MEDLINE et ProQuest, de même qu'une recherche dans la littérature grise sur les sites Web d'organisations majeures du domaine des traumas, de leur création à juin 2019, sans restriction de langue. La population à l'étude était l'ensemble des patients blessés traités en centre de traumatologie. L'intervention était l'audit du centre de traumatologie. Les groupes de comparaison correspondaient aux centres de traumatologie n'ayant pas subi d'audit, ou le même centre, avant son premier audit ou un audit subséquent. Le principal résultat à l'étude était la mortalité en milieu hospitalier; les résultats secondaires étaient les événements indésirables, l'utilisation des ressources et les processus de soins. Nous avons calculé des estimations sommaires par méta-analyse à effets aléatoires sur données groupées.

Résultats : Sur les 5125 citations retenues, 29 publications sur des études menées aux États-Unis répondaient à nos critères d'inclusion. La mortalité était le résultat le plus souvent à l'étude $(n=20)$, puis suivaient les processus de soins $(n=12)$, l'utilisation des ressources $(n=12)$ et les événements indésirables $(n=7)$. Le risque de biais était important ou critique dans 22 études. Nous avons observé une association imprécise entre l'audit et une baisse de la mortalité (risque relatif 0,74 ; intervalle de confiance à $95 \% 0,52$ à 1,06$)$ chez les patients ayant subi un trauma grave. 
Conclusion : Notre revue a conclu qu'il y avait des associations mitigées et manquant d'uniformité entre l'audit et les processus de soins ou les issues pour les patients. La validité des données à l'étude était limitée par un manque de contrôles fiables, ainsi que par l'absence de données provenant d'autres pays que les États-Unis, ce qui empêche l'extrapolation à d'autres systèmes de santé. Des études quasi expérimentales devront être menées pour évaluer les effets de l'audit des centres de traumatologie.

Enregistrement de la revue systématique : Registre PROSPERO, numéro CRD42018107083.

$\mathbf{T}$ he introduction of trauma systems, defined as an organized and multidisciplinary response to injury from prehospital care to rehabilitation and community integration, has led to important reductions in injury burdens in many high-income countries. ${ }^{1,2}$ Essential to the development of a trauma system is the designation of trauma centres according to levels of care (levels $\mathrm{I}-\mathrm{V}$ for adults, and I or II for pediatric centres), which is commonly the role of states or provinces. ${ }^{3}$ Trauma centres are acute care hospitals where resources are prioritized to ensure that injured patients receive appropriate and timely care. ${ }^{4,5}$ Injury organizations, including the American College of Surgeons, have established trauma facility standard guidelines. ${ }^{3}$ These guidelines have been used to develop trauma centre verification or accreditation processes, aimed to determine whether trauma centres are fulfilling the criteria for optimal care. "Accreditation" and "verification" of trauma centres refer to the same process; hereafter we use the term verification to refer to both. ${ }^{5,6}$

The terms "verification" and "designation" are sometimes used interchangeably despite having different meanings. ${ }^{7-10}$ Designation is conducted by regional health authorities at the local or state stage, where centres are categorized in levels (I-V for adults, and I or II for pediatric centres), whereas verification (or accreditation) is generally an optional program to verify that a facility is performing as a trauma centre and meets the criteria for its designation level. ${ }^{5,9,11}$ For example, verification is offered by the American College of Surgeons in the United States ${ }^{3,12,13}$ and accreditation by Accreditation Canada; ${ }^{6}$ these organizations are not responsible for designation. ${ }^{11} \mathrm{~A}$ centre can be designated at a particular level without having received verification. ${ }^{9}, 10$ In some US states or Canadian provinces, regulatory agencies may require regular verification for a trauma centre to maintain designation within their systems. Verification allows for standardization of personnel and equipment and a facility's commitment to trauma care. ${ }^{9}$ Perceived advantages of verification include commitment to trauma care, and identification of opportunities and priorities for improvement. ${ }^{14}$ Verification is, however, an expensive and resource-intensive process. ${ }^{15,16}$ It generally requires a centre to submit a prereview questionnaire and have an on-site visit by an experienced peer review team. ${ }^{3}$ A summary of verification modalities in different countries is presented in Table 1 .

Although verification has become a common practice, ${ }^{14,17}$ the evidence of its effectiveness on patient outcomes has not been systematically assessed and synthesized. It is essential to know whether the allocation of financial and human resources used in the verification process has its intended effect. ${ }^{17,18}$ This systematic review aims to synthesize available evidence on trauma centre verification to evaluate whether verification reduces in-hospital mortality, adverse events and resource use and improves processes of care.

\section{MethodS}

The protocol of this review was registered in the PROSPERO database (record CRD42018107083) and published. ${ }^{19}$ The review was conducted in compliance with the Preferred Reporting Items for Systematic Reviews and Meta-Analyses (PRISMA) guidelines..$^{20} \mathrm{We}$ received ethics approval for this project from the McGill University Faculty of Medicine Research Ethics Committee.

Table 1. Examples of verification agencies
\begin{tabular}{|llcc|}
\hline \multicolumn{1}{|c|}{ Agency } & $\begin{array}{c}\text { Certificate } \\
\text { duration, yr }\end{array}$ & First verification \\
\hline Jurisdiction & \multicolumn{1}{c|}{$\begin{array}{l}\text { American College of Surgeons } \\
\text { United States* }\end{array}$} & 3 & 1987 (ongoing) \\
\hline $\begin{array}{l}\text { Canada (except } \\
\text { Quebec) }\end{array}$ & Trauma Association of Canada & 5 & 1995 (to 2014) \\
\cline { 2 - 4 } Quebect & Accreditation Canada & 4 & 2014 (ongoing) \\
\hline Australia & $\begin{array}{l}\text { Institut national d'excellence en santé et } \\
\text { services sociaux }\end{array}$ & $\sim 6$ & 1995 (ongoing) \\
\hline $\begin{array}{l}\text { *Some states (e.g., Pennsylvania) have their own verification agencies. } \\
\text { tVerification is mandatory in Quebec. }\end{array}$ & & & \\
\hline
\end{tabular}




\section{Literature search and study selection}

We conducted a systematic search of the CINAHL, Embase, HealthStar, MEDLINE and ProQuest databases, as well as the websites of key injury organizations for grey literature from inception to June 2019, without language restrictions. Manual searches for additional eligible studies were performed by reviewing the reference lists of included studies. The search strategy is available in Appendix 1 (available at canjsurg.ca/016219-a1). Conference abstracts were included unless they were subsequently published as full articles.

\section{Study population and intervention}

Our study population consisted of injured patients treated at trauma centres. The intervention under evaluation was trauma centre verification. Comparison groups consisted of nonverified centres, or the same centre before it was first verified or re-verified. We considered all study designs; however, narrative studies without a quantitative estimate of the association between verification and the investigated outcomes were excluded.

\section{Outcomes}

Our primary outcome was in-hospital mortality. Secondary outcomes included population-based injury-related mortality, adverse events (e.g., complications), resource use (e.g., length of stay [LOS] and costs) and adherence to evidence-based processes of care (e.g., nonsurgical management of splenic injuries).

\section{Data collection and extraction}

After duplicates were removed from the search results, ${ }^{21}$ titles and abstracts were independently screened by 2 authors (B.B. and M.C.) using a Web and mobile app for systematic reviews. ${ }^{22}$ In case of disagreement or uncertainty, full papers were retrieved and discussed with a third author (L.M.). Full texts of selected studies were retrieved and examined to determine eligibility by 2 authors (B.B. and M.C.), who also independently extracted the data using standardized forms. When available, data recorded included country of the study, the number of centres, study design, patient demographic characteristics and outcome. Efforts were made to contact the corresponding author for further information when needed. Descriptive statistics and measures of associations were extracted directly from the studies or computed if enough information was provided. ${ }^{23-25}$

We assessed the risk of bias using the Risk Of Bias In Non-randomised Studies - of Interventions (ROBINS-I) assessment tool. ${ }^{26} \mathrm{We}$ evaluated the quality of the collective evidence and strength of recommendations using
Grading of Recommendations Assessment, Development and Evaluation (GRADE) working group methodology. ${ }^{27}$

\section{Statistical analysis}

We summarized included studies descriptively. Owing to the diverse types of measures of association used and missing standard errors (SEs) or confidence intervals (CIs), some studies were not included in the meta-analysis. These studies were summarized narratively.

For studies included in the meta-analysis, we calculated the overall summary estimates, including relative risks (RRs), odds ratios (ORs) and weighted mean difference using random-effects meta-analysis. ${ }^{28} \mathrm{We}$ quantified heterogeneity with the $I^{2}$ statistic. ${ }^{29} \mathrm{We}$ also produced funnel plots to examine the potential for publication bias. Sensitivity analysis according to the risk of bias was planned but could not be done owing to the small number of studies included. All analyses were performed with admetan and metafunnel packages in Stata 15 (Statacorp). ${ }^{30}$

\section{Results}

A total of 5125 citations were initially identified by the search strategy after de-duplication. Among them, 102 articles were selected for full-text review, of which 29 satisfied our inclusion criteria (Fig. 1 and Appendix 1, Table S1).

All included studies assessed American College of Surgeons verification in the US and were observational, including 18 cross-sectional, ${ }^{7,9,10,14,16,31-43} 10$ pre-post ${ }^{8,44-52}$ and 1 time-series $^{53}$ design. Mortality was the most commonly investigated outcome $(n=20)$, followed by processes of care $(n=12)$, resource use $(n=12)$ and adverse events $(n=7)$. A summary of study characteristics is presented in Table 2. It was not possible to compute CIs for the measure of associations in 7 studies (24\%). Almost half of included studies (13 [45\%]) did not adjust for centre or patient case mix characteristics, $8,35,38,39,40,44,46-52$ and only one-third (6/18 [33\%]) of multicentre studies considered the clustered nature of the data in their analyses. The risk of bias was serious to critical in 22 studies, and moderate in 7 (Table 3).

\section{Outcomes}

\section{Mortality}

Twenty studies ${ }^{7-10,14,16,31,33,34,37,42-45,47-51,53}$ investigated mortality, of which 18 looked at in-hospital mortality. Seven of the $20(35 \%)$ focused on pediatric injured patients, 4 $(20 \%)$ on adults, and $9(45 \%)$ on both. Most studies had cross-sectional (11) or pre-post (8) designs, with only 1 time-series study. Half of the studies presented only crude estimates.

We excluded 5 studies from the meta-analysis owing to the different effect measure scales used, as well as missing 


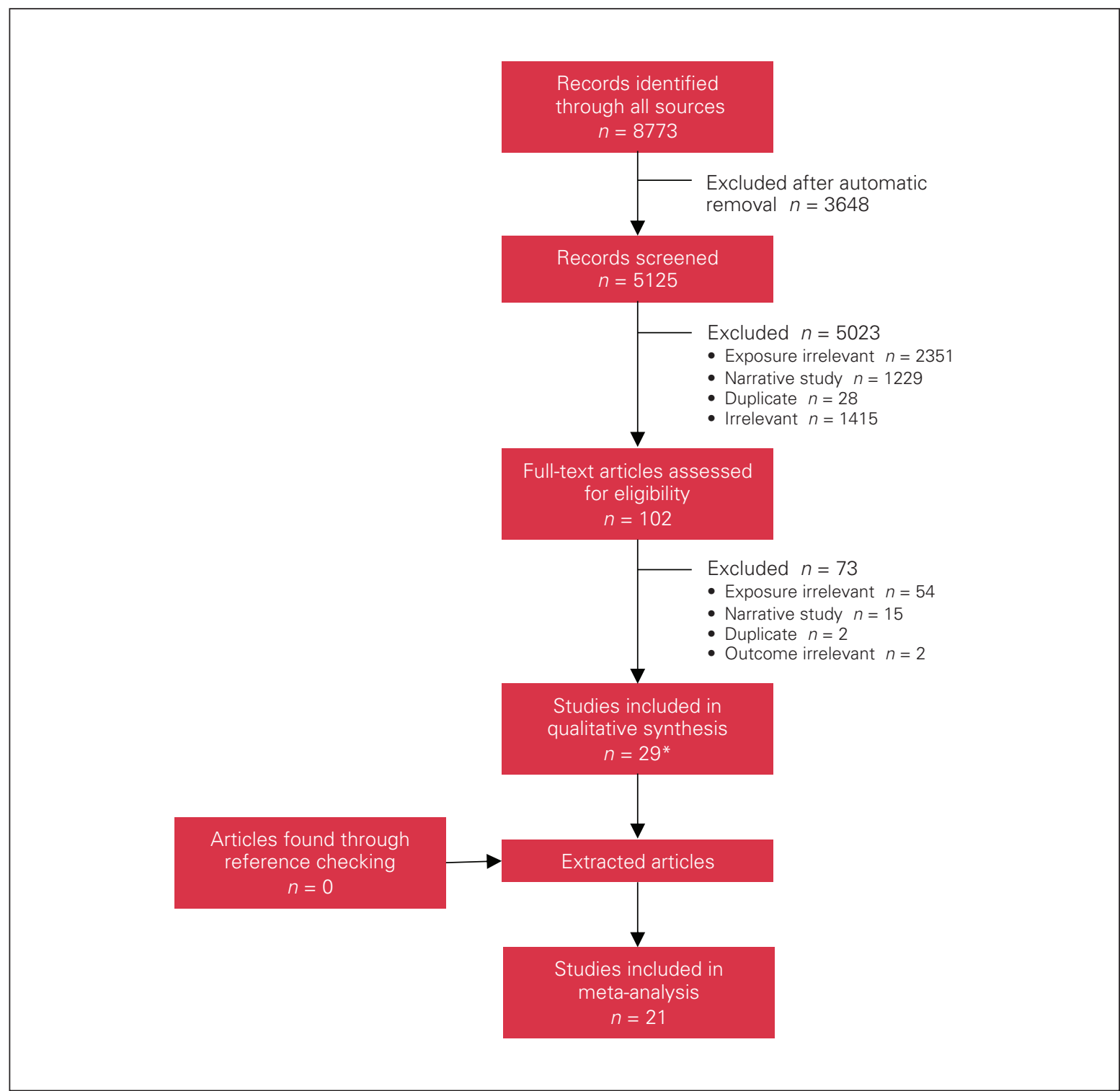

Fig. 1. Flow diagram showing study selection. ${ }^{*}$ Including 3 conference abstracts and 1 thesis.

SEs (Table 2). Jenkins and colleagues ${ }^{43}$ found that mortality increased during surgery conferences compared to nonconference dates in trauma centres lacking verification (OR 1.2, 95\% CI 1.1 to 1.4). However, among verified trauma centres, no association was observed. Piontek and colleagues $^{45}$ found a $22 \%$ reduction in standardized mortality ratio following verification of a level II trauma centre. The study by Schubert and colleagues, ${ }^{9}$ one of the few studies that accounted for time-varying verification status during the study period, showed a protective association in lower-level centres (RR $0.84,95 \%$ CI 0.72 to 0.99 for level III and RR $0.25,95 \%$ CI 0.12 to 0.54 for level IV). Notrica and colleagues ${ }^{37}$ found that population-based pediatric injury mortality rates in US states with verified level I pediatric trauma centres were $37 \%$ lower than those in states without a verified pediatric trauma centre. The only time-series analysis showed that the lagged crude rate of verified level I pediatric trauma centres was protective and contributed to a decline $(12 \%, 95 \%$ CI $4 \%$ to $18 \%)$ in the rate of change in adolescent injury mortality in the US between 1999 and 2015. ${ }^{53} \mathrm{~A}$ similar, but smaller, protective effect was observed for combined adult and pediatric verified level I trauma centres.

We included 15 studies in the meta-analysis of mortality. $7,8,10,14,16,31,33,34,42,44,47-51$ Analysis of crude RR $(n=11)$ showed that verification was generally associated with decreased mortality (Fig. 2A). This association was also observed in analysis of adjusted estimates $(n=7)$, except among severely injured patients (Injury Severity Score > 24) (OR 1.1, 95\% CI 1.05 to 1.30) (Fig. 2B).

Funnel plots indicated a certain degree of asymmetry, which was more pronounced among studies providing crude estimates (Fig. 3A and Fig. 3B), which suggests publication bias. These figures also showed substantial 
Table 2 (part 1 of 3). Summary of characteristics of included studies

\begin{tabular}{|c|c|c|c|c|c|}
\hline Design; study & $\begin{array}{l}\text { Outcome(s) } \\
\text { investigated }\end{array}$ & $\begin{array}{c}\text { Population/age, } \\
\text { yr }\end{array}$ & $\begin{array}{l}\text { No. of centres } \\
\text { (patients) }\end{array}$ & $\begin{array}{l}\text { Data } \\
\text { collection } \\
\text { period }\end{array}$ & Summary of results \\
\hline \multicolumn{6}{|l|}{ Cross-sectional } \\
\hline Osler et al., ${ }^{31} 2001$ & Mortality & $\begin{array}{l}\text { Pediatrics (all } \\
\text { injuries) } /<18\end{array}$ & 53 (49 428) & 1985-1996 & $\begin{array}{l}\text { Survival rates among children at verified centres were higher } \\
\text { than at nonverified centres (OR } 0.75,95 \% \mathrm{Cl} 0.58 \text { to } 0.97 \text { ). } \\
\text { However, authors could not conclude that it was the process of } \\
\text { verification itself that improved outcomes. It is possible that } \\
\text { only trauma centres with better results pursue verification. If } \\
\text { so, verification would recognize, but not contribute to, improved } \\
\text { outcomes. }\end{array}$ \\
\hline $\begin{array}{l}\text { Hesdorffer et al., } \\
2002\end{array}$ & $\begin{array}{l}\text { Processes of } \\
\text { care }\end{array}$ & $\begin{array}{l}\text { Adults }(\mathrm{TBI}) / \mathrm{no} \\
\text { details }\end{array}$ & 411 (no details) & 1999-2000 & $\begin{array}{l}\text { Authors surveyed all designated US trauma centres caring } \\
\text { for adults with severe TBI to determine degree of } \\
\text { guideline compliance and identify predictors. Full } \\
\text { compliance occurred more commonly among hospitals } \\
\text { with level I designation, a neurosurgery residency } \\
\text { program, treatment protocols, a neurologic ICU and ACS } \\
\text { verification ( } 23 \% \text { v. } 15 \%) \text {. }\end{array}$ \\
\hline $\begin{array}{l}\text { Demetriades et } \\
\text { al., }{ }^{33} 2006\end{array}$ & Mortality & $\begin{array}{l}\text { Adults (all } \\
\text { severe injuries } \\
[I S S>15]) />14\end{array}$ & 256 (130 154) & 1994-2003 & $\begin{array}{l}\text { Authors compared verified centres and nonverified centres and } \\
\text { found that adjusted mortality in nonverified centres was higher } \\
\text { than in verified level I centres (OR } 1.09,95 \% \mathrm{Cl} 1.05 \text { to 1.13). } \\
\text { However, they highlighted that this finding needs cautious } \\
\text { interpretation because the group of nonverified centres } \\
\text { included facilities that were only state-designated and those } \\
\text { with no trauma centre designation. }\end{array}$ \\
\hline $\mathrm{Kim}^{34} 2006$ & $\begin{array}{l}\text { Mortality, } \\
\text { processes of } \\
\text { care, resources }\end{array}$ & $\begin{array}{l}\text { All patients } \\
\text { (head injuries)/ } \\
\leq 89\end{array}$ & $16(487)$ & 2002-2003 & $\begin{array}{l}12 \text { verified centres and } 4 \text { state-designated centres. No } \\
\text { associations between verification and outcomes investigated } \\
\text { (mortality, LOS, home discharge disposition and time to } \\
\text { surgery). }\end{array}$ \\
\hline $\begin{array}{l}\text { Hesdorffer et al., } \\
2007\end{array}$ & $\begin{array}{l}\text { Processes of } \\
\text { care }\end{array}$ & $\begin{array}{l}\text { Adults (TBI)/no } \\
\text { details }\end{array}$ & 413 (no details) & 2006 & $\begin{array}{l}\text { Web-based survey conducted in } 413 \text { designated trauma } \\
\text { centres admitting patients with severe TBI. Good adherence } \\
\text { was defined as adherence to median number of guidelines. } \\
\text { Higher rate of good adherence was found in verified trauma } \\
\text { centres }(70.6 \%, n=153) \text { than in state-designated centres } \\
(60.8 \%, n=232) \text {. }\end{array}$ \\
\hline $\begin{array}{l}\text { Horton et al., } \\
2008\end{array}$ & $\begin{array}{l}\text { Processes of } \\
\text { care, resources }\end{array}$ & $\begin{array}{l}\text { All patients (all } \\
\text { injuries)/no } \\
\text { details }\end{array}$ & 156 (no details) & 2005 & $\begin{array}{l}\text { Authors surveyed } 435 \text { trauma centres (level I and II) throughout } \\
\text { the US. } 156 \text { surveys were returned. ACS verification and } \\
\text { trauma level I designation were independent predictors of } \\
\text { recombinant factor VIla use. }\end{array}$ \\
\hline $\begin{array}{l}\text { Smith et al., }{ }^{10} \\
2011\end{array}$ & $\begin{array}{l}\text { Mortality, } \\
\text { adverse events }\end{array}$ & $\begin{array}{l}\text { All patients (all } \\
\text { injuries)/> } 16\end{array}$ & $\begin{array}{l}\text { No details } \\
(519402)\end{array}$ & 2002-2006 & $\begin{array}{l}\text { Authors compared verified level I facilities to state- } \\
\text { designated centres. Overall, no adjusted survival advantage. } \\
\text { However, among patients with acute respiratory distress } \\
\text { syndrome, mortality rate was lower after admission to } \\
\text { ACS-verified centres than to state-designated centres } \\
(20.3 \% \text { v. } 27.1 \%) \text {. }\end{array}$ \\
\hline $\begin{array}{l}\text { Theologis et al., } \\
2012\end{array}$ & $\begin{array}{l}\text { Processes of } \\
\text { care }\end{array}$ & $\begin{array}{l}\text { All patients } \\
\text { (spine injuries)/ } \\
\text { no details }\end{array}$ & No details & No details & $\begin{array}{l}\text { Authors contacted trauma managers in all level I trauma } \\
\text { centres in US to analyze institutions' official cervical spine } \\
\text { clearance protocols, if applicable. Response rate was } 83 \% \text {. } \\
\text { Two-thirds of respondents had an official cervical spine } \\
\text { clearance protocol. More ACS-verified centres than } \\
\text { nonverified centres had protocols ( } 75 \% \text { v. } 54 \% \text { ). }\end{array}$ \\
\hline $\begin{array}{l}\text { Notrica et al., } \\
2012\end{array}$ & Mortality & $\begin{array}{l}\text { Pediatrics (all } \\
\text { injuries) } /<18\end{array}$ & NA & 2008 & $\begin{array}{l}\text { Population-based study of pediatric injury mortality rates } \\
\text { per } 100000 \text {. Authors determined availability of verified } \\
\text { PTCs and verified ATCs in each state and compared } \\
\text { mortality rates. Findings highlight protective association } \\
\text { between pediatric injury mortality rates and presence of } \\
\text { verified level I PTCs. }\end{array}$ \\
\hline $\begin{array}{l}\text { Brown et al., }{ }^{16} \\
2013\end{array}$ & Mortality & $\begin{array}{l}\text { Adults (all } \\
\text { injuries)/> } 15\end{array}$ & 374 (900 274) & 2007-2008 & $\begin{array}{l}\text { Retrospective analysis of } 246 \text { verified and } 128 \text { state- } \\
\text { designated centres. Verified level I centres had lower median } \\
\text { SMR than state-designated centres (0.95 [IOR 0.82-1.05] v. } \\
1.02 \text { [IQR 0.87-1.15]); no difference in level II centres. } \\
\text { State-designated level II centres had higher SMR outliers than } \\
\text { ACS-verified level II centres. }\end{array}$ \\
\hline $\begin{array}{l}\text { Russell et al., }{ }^{40} \\
2015\end{array}$ & Resources & $\begin{array}{l}\text { Pediatrics (all } \\
\text { injuries)/no } \\
\text { details }\end{array}$ & 102 (no details) & No details & $\begin{array}{l}\text { Authors conducted structured telephone survey of } \\
\text { emergency departments registered with National } \\
\text { Association of Children's Hospitals and ACS-verified PTCs. } \\
\text { Bedside ultrasonography has become largely ubiquitous for } \\
\text { care of children at designated PTCs; no significant difference } \\
\text { between verified PTCs ( } 97 \% \text { [56/58]) and designated trauma } \\
\text { centres (89\% [39/44]). }\end{array}$ \\
\hline
\end{tabular}


Table 2 (part 2 of 3). Summary of characteristics of included studies

\begin{tabular}{|c|c|c|c|c|c|}
\hline Design; study & $\begin{array}{l}\text { Outcome(s) } \\
\text { investigated }\end{array}$ & $\begin{array}{c}\text { Population/age, } \\
y r\end{array}$ & $\begin{array}{l}\text { No. of centres } \\
\text { (patients) }\end{array}$ & $\begin{array}{l}\text { Data } \\
\text { collection } \\
\text { period }\end{array}$ & Summary of results \\
\hline $\begin{array}{l}\text { Alarhayem et al., } \\
2015\end{array}$ & $\begin{array}{l}\text { Processes of } \\
\text { care }\end{array}$ & $\begin{array}{l}\text { Pediatrics } \\
\text { (splenic } \\
\text { injuries) } /<17\end{array}$ & $\begin{array}{c}\text { No details } \\
\text { (2342) }\end{array}$ & 2012 & $\begin{array}{l}\text { Majority of children with splenic injuries were treated in } \\
\text { nonverified PTCs. Verified level I PTCs had highest success } \\
\text { with nonoperative management of high-grade splenic injuries } \\
(6 \%) \text {, followed by ACS-verified level II PTCs (10\%) and } \\
\text { nonverified PTCs (13\%). }\end{array}$ \\
\hline $\begin{array}{l}\text { Bogumil et al., }{ }^{41} \\
2017\end{array}$ & $\begin{array}{l}\text { Processes of } \\
\text { care }\end{array}$ & $\begin{array}{l}\text { Pediatrics (all } \\
\text { injuries) } /<18\end{array}$ & $\begin{array}{l}\text { No details } \\
(475527)\end{array}$ & 2007-2014 & $\begin{array}{l}\text { Authors used National Trauma Data Bank to compare } \\
\text { prevalence of nonaccidental trauma between ACS-verified } \\
\text { PTCs and. They calculated crude and ISS-adjusted prevalence } \\
\text { ratio estimates. Prevalence of nonaccidental trauma was higher } \\
\text { at ACS-verified PTCs than at non-ACS-verified PTCs. }\end{array}$ \\
\hline $\begin{array}{l}\text { Grossman et al., }{ }^{14} \\
2017\end{array}$ & $\begin{array}{l}\text { Mortality, } \\
\text { adverse events }\end{array}$ & $\begin{array}{l}\text { All patients (all } \\
\text { injuries)/all } \\
\text { ages }\end{array}$ & 94 (392 997) & 2012 & $\begin{array}{l}\text { Authors analyzed national representative sample of } 94 \text { trauma } \\
\text { centres ( } 72 \text { verified and } 22 \text { nonverified). Measurable benefits in } \\
\text { complications were observed only among cases of major } \\
\text { trauma (ISS }>25 \text { ) in all age groups. }\end{array}$ \\
\hline $\begin{array}{l}\text { Roubik et al., }{ }^{42} \\
2017\end{array}$ & Mortality & $\begin{array}{l}\text { All patients } \\
\text { (ground-level } \\
\text { falls)/> } 15\end{array}$ & 794 (812 051) & 2007-2014 & $\begin{array}{l}\text { Retrospective analyses comparing } 335 \text { verified and } 459 \\
\text { state-designated centres. SMR was lowest for verified } \\
\text { level III/IV centres }(0.97,95 \% \mathrm{Cl} 0.97 \text { to } 0.98) \text { and highest } \\
\text { for state-designated level III/IV centres }(1.04,95 \% \mathrm{Cl} 1.04 \\
\text { to } 1.04) \text {. }\end{array}$ \\
\hline $\begin{array}{l}\text { Agrawal et al., } \\
2018\end{array}$ & $\begin{array}{l}\text { Mortality, } \\
\text { adverse events, } \\
\text { resources }\end{array}$ & $\begin{array}{l}\text { All patients (all } \\
\text { injuries) } /<16\end{array}$ & 109 (1 504 848) & $\begin{array}{l}2002-2009 \\
2013-2014\end{array}$ & $\begin{array}{l}\text { After risk adjustment, authors observed lower mean ICU LOS } \\
(-0.2 \mathrm{~d} \text { [SD } 0.02 \mathrm{~d}]) \text {, hospital LOS }(-0.3 \mathrm{~d} \text { [SD } 0.019 \mathrm{~d}]) \text {, } \\
\text { mortality (OR } 0.94,95 \% \mathrm{Cl} 0.92 \text { to } 0.96) \text { and number of } \\
\text { patients who developed complications at verified centres than } \\
\text { at state-designated centres. }\end{array}$ \\
\hline $\begin{array}{l}\text { Schubert et al., }{ }^{9} \\
2019\end{array}$ & $\begin{array}{l}\text { Mortality, } \\
\text { adverse events }\end{array}$ & $\begin{array}{l}\text { Adults (all } \\
\text { injuries)/> } 17\end{array}$ & 863 (4 044 449) & 2010-2015 & $\begin{array}{l}\text { Overall, patients admitted to verified v. state-designated } \\
\text { centres had similar adjusted mortality risk (RR } 1.00,95 \% \mathrm{Cl} \\
0.91 \text { to } 1.03 \text { ) and unplanned return to operating room (RR } 1.10 \text {, } \\
95 \% \mathrm{Cl} 0.92 \text { to } 1.31 \text { ), but higher unplanned intubation (RR 1.30, } \\
95 \% \mathrm{Cl} 1.11 \text { to 1.52). However, verified level III and IV facilities } \\
\text { had lower adjusted mortality risk, with much lower mortality } \\
\text { risk in ACS-verified level IV facilities. }\end{array}$ \\
\hline $\begin{array}{l}\text { Jenkins et al., } \\
2019\end{array}$ & Mortality & $\begin{array}{l}\text { Adults (all } \\
\text { injuries)/> } 16\end{array}$ & 155 (94 655) & 2010-2011 & $\begin{array}{l}\text { Authors examined association between national surgery } \\
\text { conferences and in-hospital trauma mortality. Mortality } \\
\text { increased significantly during meetings among patients } \\
\text { admitted to hospitals that lacked ACS trauma verification; } \\
\text { association was particularly pronounced among patients with } \\
\text { penetrating injuries. }\end{array}$ \\
\hline \multicolumn{6}{|l|}{ Pre-post } \\
\hline $\begin{array}{l}\text { Richardson et al., } \\
1997\end{array}$ & $\begin{array}{l}\text { Mortality, } \\
\text { processes of } \\
\text { care }\end{array}$ & $\begin{array}{l}\text { All patients (all } \\
\text { injuries)/no } \\
\text { details }\end{array}$ & $2(381)$ & $\begin{array}{l}1988 \text { and } \\
1995\end{array}$ & $\begin{array}{l}\text { Two level III facilities; } 1 \text { had received level III verification, } \\
\text { and the other had changes that lessened general surgeons' } \\
\text { involvement in initial evaluation and treatment. Verified } \\
\text { centre had increase in patients transferred to level I hospital } \\
\text { and in patient acuity. More operations were performed } \\
\text { locally, and care was delivered more efficiently. The other } \\
\text { hospital had large increase in transfers and decreased } \\
\text { admissions locally as general surgical involvement } \\
\text { decreased. }\end{array}$ \\
\hline $\begin{array}{l}\text { Piontek et al.., } \\
2003\end{array}$ & $\begin{array}{l}\text { Mortality, } \\
\text { adverse events, } \\
\text { resources }\end{array}$ & $\begin{array}{l}\text { All patients (all } \\
\text { injuries)/no } \\
\text { details }\end{array}$ & $1(7811)$ & 1993-2001 & $\begin{array}{l}\text { Pre-post study with internal and external negative control } \\
\text { outcomes of level II centre. Results suggest that efforts and } \\
\text { resources consumed in achieving ACS level II trauma centre } \\
\text { verification result in decreased LOS (by 10\%), reduced } \\
\text { in-hospital mortality (severity-adjusted mortality observed/ } \\
\text { expected ratio } 0.81 \text { before v. } 0.59 \text { after) and reduced costs (by } \\
5 \% \text { ). }\end{array}$ \\
\hline $\begin{array}{l}\text { Ehrlich et al., }{ }^{46} \\
2005\end{array}$ & $\begin{array}{l}\text { Processes of } \\
\text { care }\end{array}$ & $\begin{array}{l}\text { Pediatrics (all } \\
\text { injuries) } /<16\end{array}$ & 1 (no details) & 1997-2002 & $\begin{array}{l}\text { Verification process at a level I ATC seeking level I PTC } \\
\text { verification affected patient care through changes in care } \\
\text { indicators. Mortality and ISS distributions remained } \\
\text { unaltered. Patient evaluation, including radiology and time to } \\
\text { discharge from emergency department ( }<120 \text { min), } \\
\text { improved. PICU duration of stay increased, and prehospital } \\
\text { and emergency department fluid monitoring remained } \\
\text { unsatisfactory. }\end{array}$ \\
\hline $\begin{array}{l}\text { Maggio et al., }{ }^{47} \\
2009\end{array}$ & $\begin{array}{l}\text { Mortality, } \\
\text { resources }\end{array}$ & $\begin{array}{l}\text { All patients (all } \\
\text { injuries)/no } \\
\text { details }\end{array}$ & 1 (3891) & $\begin{array}{l}2001 \text { and } \\
2007\end{array}$ & $\begin{array}{l}\text { Commitment to ACS verification resulted in increased } \\
\text { admissions and interfacility transfers. Despite admission of } \\
\text { more seriously injured patients, there was sustained } \\
\text { reduction in mortality (by } 47 \% \text { in patients with ISS > 24) and } \\
\text { trend toward decreased ICU LOS. Authors also found 78\% } \\
\text { increase in revenue and sustained increase in hospital } \\
\text { profitability. }\end{array}$ \\
\hline
\end{tabular}


Table 2 (part 3 of 3). Summary of characteristics of included studies

\begin{tabular}{|c|c|c|c|c|c|}
\hline Design; study & $\begin{array}{l}\text { Outcome(s) } \\
\text { investigated }\end{array}$ & $\begin{array}{c}\text { Population/age, } \\
y r\end{array}$ & $\begin{array}{l}\text { No. of centres } \\
\text { (patients) }\end{array}$ & $\begin{array}{l}\text { Data } \\
\text { collection } \\
\text { period }\end{array}$ & Summary of results \\
\hline $\begin{array}{l}\text { Norwood et al., }{ }^{48} \\
2011\end{array}$ & $\begin{array}{l}\text { Mortality, } \\
\text { resources }\end{array}$ & $\begin{array}{l}\text { All patients } \\
\text { (major torso } \\
\text { vascular } \\
\text { injuries)/no } \\
\text { details }\end{array}$ & $1(274)$ & 1992-2008 & $\begin{array}{l}\text { Centre was verified level II facility before obtaining level I } \\
\text { verification in 1998. Commitment of hospital resources required } \\
\text { to achieve level I verification in community hospital improved } \\
\text { survival, particularly in patients with blunt or penetrating } \\
\text { thoracic injuries ( } 73 \% \text { before v. } 30 \% \text { after). }\end{array}$ \\
\hline $\begin{array}{l}\text { Murphy et al. }{ }^{49} \\
2015\end{array}$ & $\begin{array}{l}\text { Mortality, } \\
\text { processes of } \\
\text { care, resources }\end{array}$ & $\begin{array}{l}\text { Pediatrics } \\
\text { (splenic } \\
\text { injuries) } /<16\end{array}$ & No details (231) & 1998-2012 & $\begin{array}{l}\text { Addition of verified PTC within inclusive trauma system was } \\
\text { associated with significant reduction in proportion of patients } \\
\text { undergoing splenectomy. However, results regarding mortality } \\
\text { were inconclusive (RR } 0.70,95 \% \mathrm{Cl} 0.12 \text { to } 4.09 \text { ). }\end{array}$ \\
\hline Choi et al., ${ }^{8} 2016$ & $\begin{array}{l}\text { Mortality, } \\
\text { adverse events, } \\
\text { resources }\end{array}$ & $\begin{array}{l}\text { Pediatrics (all } \\
\text { injuries)/no } \\
\text { details }\end{array}$ & $1(4353)$ & $\begin{array}{l}2009-2010 \\
\text { and } \\
2012-2014\end{array}$ & $\begin{array}{l}\text { Retrospective review of state-designated level I PTC, } \\
\text { comparing } 2 \text { years before and } 2 \text { years after verification. Overall, } \\
\text { no differences in mean age or ISS. Hospital and PICU LOS, } \\
\text { ventilator days and mortality were also unchanged. Proportion } \\
\text { of PICU admissions decreased from } 17.2 \% \text { to } 13.7 \% \text {. Adverse } \\
\text { events in form of hospital-acquired conditions also decreased } \\
\text { following verification, most notably through reduction in } \\
\text { pneumonia. }\end{array}$ \\
\hline $\begin{array}{l}\text { Schlegel et al., }{ }^{51} \\
2018\end{array}$ & $\begin{array}{l}\text { Mortality, } \\
\text { resources }\end{array}$ & $\begin{array}{l}\text { Pediatrics (all } \\
\text { injuries) } /<18\end{array}$ & $1(1190)$ & 2004-2016 & $\begin{array}{l}\text { Retrospective analysis divided into } 3 \text { chronologic treatment } \\
\text { eras: early ATC, PTC and late PTC after ACS verification. } \\
\text { Decrease in intensive care admissions was identified during } \\
\text { late PTC compared to early PTC and ATC ( } 51 \% \text { v. } 62.4 \% \text { v. } \\
67 \%) \text {, but overall mortality was unchanged. }\end{array}$ \\
\hline $\begin{array}{l}\text { Alexander et al., } \\
2019\end{array}$ & $\begin{array}{l}\text { Mortality, } \\
\text { adverse events, } \\
\text { processes of } \\
\text { care, resources }\end{array}$ & $\begin{array}{l}\text { Pediatrics } \\
\text { (splenic } \\
\text { injuries) } /<18\end{array}$ & $1(126)$ & 2005-2017 & $\begin{array}{l}\text { Comparison of verified level I ATC after PTC verification. Lower } \\
\text { rate of splenic intervention under PTC than ATC verification } \\
\text { ( } 7.1 \% \text { v. } 19.6 \% \text { ). Primary driver of decrease was reduction in } \\
\text { operative rates ( } 14.3 \% \text { under ATC v. } 4.3 \% \text { under PTC). Average } \\
\text { hospital LOS ( } 7.4 \mathrm{~d} \text { v. } 6.5 \mathrm{~d} \text { ) and average ICU LOS ( } 2.7 \mathrm{~d} \text { v. } 2.3 \\
\text { d) were similar. No change in in-hospital mortality. }\end{array}$ \\
\hline $\begin{array}{l}\text { Abd El-Shafy et } \\
\text { al., }^{52} 2019\end{array}$ & $\begin{array}{l}\text { Processes of } \\
\text { care, resources }\end{array}$ & $\begin{array}{l}\text { Pediatrics (all } \\
\text { injuries)/no } \\
\text { details }\end{array}$ & 1 (1293) & $\begin{array}{l}2011 \text { and } \\
2016\end{array}$ & $\begin{array}{l}\text { Process changes associated with ACS level I pediatric } \\
\text { verification and reductions in nonsurgical admission rate ( } 29 \% \\
\text { in } 2011 \text { v. } 5 \% \text { in } 2016 \text { ) were also marked by reduction in } \\
\text { hospital LOS ( } 3.78 \text { d v. } 3 \text { d). }\end{array}$ \\
\hline \multicolumn{6}{|l|}{ Time-series } \\
\hline $\begin{array}{l}\text { Notrica et al., }{ }^{53} \\
2018\end{array}$ & Mortality & $\begin{array}{l}\text { Pediatrics (all } \\
\text { injuries)/15-17 }\end{array}$ & NA & 1999-2015 & $\begin{array}{l}\text { Authors collected prospective data on motor vehicle fatalities, } \\
\text { crash characteristics, state driving laws and verified trauma } \\
\text { centres for all } 50 \text { US states. Verified PTCs were associated } \\
\text { with } 12 \% \text { decrease in rate of change in crude adolescent } \\
\text { fatalities. }\end{array}$ \\
\hline
\end{tabular}

variability among studies with larger samples. The GRADE results suggest that the quality of evidence is very low (Appendix 1, Supplemental Table S2).

\section{Resource use}

Length of stay, including overall and intensive care unit, was the most studied outcome in the resource use category (10 of 12 studies)..$^{7,8,34,45,47-52}$ Other outcomes examined were blood products transfused, hospital charges, mechanical ventilation, bedside use of ultrasonography and use of recombinant factor VIIa (rFVIIa). ${ }^{36,40}$ Six of 12 studies focused on injured pediatric patients. Only 4 studies adjusted for at least 1 potential confounder. , $34,36,45^{2}$

We conducted a meta-analysis of 7 studies assessing LOS. Three studies described the distribution of LOS using the median and interquartile range, ${ }^{49-51}$ and 4 presented the mean and standard deviation. ${ }^{8,34,45-48} \mathrm{We}$ used a well-established technique to combine results reported on log-transformed or raw scales ${ }^{24,25}$ to conduct the analysis. Because of the skewed distribution of LOS, we computed only weighted mean differences of the log-transformed LOS (which can be interpreted as the geometric mean ratio when exponentiated). Our results suggest that American College of Surgeons verification was associated with longer intensive care unit LOS but not hospital LOS (Fig. 4).

Funnel plots displayed asymmetry in favour of studies with increased LOS among verified centres (Fig. 5). Our GRADE assessment suggested that the evidence was of very low quality (Appendix 1, Table S2).

Studies excluded from meta-analysis showed mixed and inconsistent results concerning the association between verification and use of various resources. For instance, Alexander and colleagues ${ }^{50}$ found that pediatric verification was associated with a decrease in the average number of blood products transfused per patient (7.2 units v. 2.4 units, 
Table 3. Risk of bias as assessed with the Risk Of Bias In Non-randomised Studies - of Interventions assessment tool

\begin{tabular}{|c|c|c|c|c|c|c|c|c|c|}
\hline Study & Confounding & $\begin{array}{l}\text { Selection of } \\
\text { participants } \\
\text { into study }\end{array}$ & $\begin{array}{l}\text { Classification } \\
\text { of } \\
\text { interventions }\end{array}$ & $\begin{array}{l}\text { Deviations } \\
\text { from } \\
\text { intended of } \\
\text { interventions }\end{array}$ & $\begin{array}{l}\text { Missing } \\
\text { data }\end{array}$ & $\begin{array}{l}\text { Measurement } \\
\text { of outcomes }\end{array}$ & $\begin{array}{l}\text { Selection of } \\
\text { reported } \\
\text { result }\end{array}$ & Overall bias & Direction \\
\hline Osler et al.,. 2011 & Serious & Serious & Serious & Serious & Serious & Low & Low & Serious & Unpredictable \\
\hline $\begin{array}{l}\text { Hesdorffer et al., }{ }^{32} \\
2002\end{array}$ & Serious & Serious & Serious & $\begin{array}{l}\text { No } \\
\text { information }\end{array}$ & Serious & Moderate & Serious & Serious & Unpredictable \\
\hline $\begin{array}{l}\text { Demetriades et al.., } \\
2006\end{array}$ & Critical & Serious & Serious & $\begin{array}{l}\text { No } \\
\text { information }\end{array}$ & $\begin{array}{l}\text { No } \\
\text { information }\end{array}$ & Low & Moderate & Serious & $\begin{array}{l}\text { Favours } \\
\text { experimental }\end{array}$ \\
\hline $\mathrm{Kim}_{,}^{34} 2006$ & Moderate & Serious & Moderate & Moderate & Critical & Low & Low & Moderate & Unpredictable \\
\hline $\begin{array}{l}\text { Hesdorffer et al., } 35 \\
2007\end{array}$ & Critical & Serious & Critical & Low & Serious & Moderate & Serious & Serious & Unpredictable \\
\hline Horton et al., ${ }^{36} 2008$ & Critical & Critical & Moderate & $\begin{array}{l}\text { No } \\
\text { information }\end{array}$ & Critical & Critical & Moderate & Critical & Unpredictable \\
\hline Smith et al.., ${ }^{10} 2011$ & Serious & Serious & Moderate & Moderate & $\begin{array}{l}\text { No } \\
\text { information }\end{array}$ & Low & Serious & Serious & Unpredictable \\
\hline $\begin{array}{l}\text { Theologis et al., }{ }^{38} \\
2012\end{array}$ & Critical & Serious & Serious & $\begin{array}{l}\text { No } \\
\text { information }\end{array}$ & $\begin{array}{l}\text { No } \\
\text { information }\end{array}$ & Moderate & $\begin{array}{l}\text { No } \\
\text { information }\end{array}$ & Critical & Unpredictable \\
\hline Notrica et al.., 2012 & Critical & Serious & Moderate & $\begin{array}{l}\text { No } \\
\text { information }\end{array}$ & $\begin{array}{l}\text { No } \\
\text { information }\end{array}$ & Moderate & Moderate & Serious & Unpredictable \\
\hline Brown et al., ${ }^{16} 2013$ & Moderate & Serious & Moderate & Moderate & Moderate & Low & Moderate & Moderate & Toward the null \\
\hline Russell et al., ${ }^{40} 2015$ & Critical & Critical & Serious & $\begin{array}{l}\text { No } \\
\text { information }\end{array}$ & $\begin{array}{l}\text { No } \\
\text { information }\end{array}$ & Critical & Moderate & Critical & Unpredictable \\
\hline $\begin{array}{l}\text { Alarhayem et al., }{ }^{39} \\
2015\end{array}$ & Critical & Critical & Moderate & $\begin{array}{l}\text { No } \\
\text { information }\end{array}$ & $\begin{array}{l}\text { No } \\
\text { information }\end{array}$ & Low & Serious & Critical & Unpredictable \\
\hline $\begin{array}{l}\text { Bogumil et al., } \\
2017\end{array}$ & Critical & Moderate & Moderate & $\begin{array}{l}\text { No } \\
\text { information }\end{array}$ & Serious & Moderate & Low & Serious & Unpredictable \\
\hline $\begin{array}{l}\text { Grossman et al., }{ }^{14} \\
2017\end{array}$ & Serious & Serious & Serious & $\begin{array}{l}\text { No } \\
\text { information }\end{array}$ & $\begin{array}{l}\text { No } \\
\text { information }\end{array}$ & Low & Moderate & Serious & Unpredictable \\
\hline Roubik et al., ${ }^{42} 2017$ & Moderate & Serious & Moderate & Serious & Critical & Low & Moderate & Serious & Unpredictable \\
\hline Agrawal et al.,7 2018 & Serious & Moderate & Low & $\begin{array}{l}\text { No } \\
\text { information }\end{array}$ & Serious & Moderate & Serious & Moderate & Unpredictable \\
\hline $\begin{array}{l}\text { Schubert et al., }{ }^{9} \\
2019\end{array}$ & Moderate & Moderate & Moderate & Low & $\begin{array}{l}\text { No } \\
\text { information }\end{array}$ & Low & Moderate & Moderate & Unpredictable \\
\hline Jenkins et al., 2019 & Low & Moderate & Low & $\begin{array}{l}\text { No } \\
\text { information }\end{array}$ & Serious & Moderate & Serious & Serious & Unpredictable \\
\hline $\begin{array}{l}\text { Richardson et al., }{ }^{44} \\
1997\end{array}$ & Critical & Low & Low & Moderate & $\begin{array}{l}\text { No } \\
\text { information }\end{array}$ & Moderate & Serious & Serious & Unpredictable \\
\hline Piontek et al., ${ }^{45} 2003$ & Moderate & Low & Low & Low & $\begin{array}{l}\text { No } \\
\text { information }\end{array}$ & Moderate & Moderate & Moderate & Unpredictable \\
\hline Ehrlich et al., ${ }^{46} 2005$ & Critical & Low & Low & Serious & $\begin{array}{l}\text { No } \\
\text { information }\end{array}$ & Moderate & Low & Serious & Unpredictable \\
\hline Maggio et al., ${ }^{47} 2009$ & Critical & Moderate & Moderate & Moderate & $\begin{array}{l}\text { No } \\
\text { information }\end{array}$ & Serious & Serious & Serious & $\begin{array}{l}\text { Favours } \\
\text { experimental }\end{array}$ \\
\hline $\begin{array}{l}\text { Norwood et al., }{ }^{48} \\
2011\end{array}$ & Critical & Low & Low & Moderate & $\begin{array}{l}\text { No } \\
\text { information }\end{array}$ & Low & Moderate & Serious & $\begin{array}{l}\text { Favours } \\
\text { experimental }\end{array}$ \\
\hline Murphy et al., ${ }^{49} 2015$ & Critical & Low & Low & Moderate & $\begin{array}{l}\text { No } \\
\text { information }\end{array}$ & Moderate & Moderate & Serious & Unpredictable \\
\hline Choi et al., 82016 & Critical & Low & Moderate & Moderate & $\begin{array}{l}\text { No } \\
\text { information }\end{array}$ & Low & Moderate & Serious & $\begin{array}{l}\text { Favours } \\
\text { Comparator }\end{array}$ \\
\hline $\begin{array}{l}\text { Schlegel et al., } \\
2018 \neq\end{array}$ & Critical & Low & Low & Moderate & $\begin{array}{l}\text { No } \\
\text { information }\end{array}$ & Moderate & Moderate & Serious & $\begin{array}{l}\text { Favours } \\
\text { experimental }\end{array}$ \\
\hline $\begin{array}{l}\text { Alexander et al., }{ }^{50} \\
2019\end{array}$ & Serious & Low & Low & Low & $\begin{array}{l}\text { No } \\
\text { information }\end{array}$ & Moderate & Serious & Moderate & $\begin{array}{l}\text { Favours } \\
\text { experimental }\end{array}$ \\
\hline $\begin{array}{l}\text { Abd El-Shafy et al., }{ }^{52} \\
2019\end{array}$ & Critical & Low & Low & Moderate & $\begin{array}{l}\text { No } \\
\text { information }\end{array}$ & Moderate & Moderate & Serious & Unpredictable \\
\hline Notrica et al.. ${ }^{53} 2018$ & Moderate & Moderate & Moderate & $\begin{array}{l}\text { No } \\
\text { information }\end{array}$ & Serious & Low & Moderate & Moderate & Unpredictable \\
\hline
\end{tabular}

$95 \%$ CI -10.1 to 0.6$)$ and in professional charges (US-\$16 171, 95\% CI $-\$ 30898$ to $-\$ 1362$ ). However, Piontek and colleagues ${ }^{45}$ reported that, after verification of a level II trauma centre, there was an increase in ventilation use (RR 1.30, 95\% CI 1.12 to 1.51 ). Horton and colleagues ${ }^{36}$ surveyed level I and II trauma centres and found that American College of Surgeons verification was a predictor of rFVIIa use (OR 3.74, 95\% CI 1.53 to 9.09) (Table 2). 
A Population; study All patients Murphy et al., 492015 Murphy et al., 2015 Roubik et al., 42017 Schubert et al., ${ }^{9} 201$ Subgroup $\left(/^{2}=0.0 \%\right)$

Severe injuries Demetriades et al., ${ }^{33} 2006$ ISS > 15 Maggio et al.,${ }^{47} 2009$ ISS $>24$ Choi et al., 2016 ISS > 15 Subgroup $\left(/^{2}=96.1 \%\right)$

Level I

Demitriades et al., ${ }^{33} 2006$

Norwood et al., ${ }^{48} 2011$

Smith et a

Smith et al., 2011

Alexander et al., ${ }^{50} 2019$

Schlegel et al., ${ }^{5 i} 2018$

Agrawal et al., ${ }^{7} 2018$

Subgroup $\left(/^{2}=95.7 \%\right)$

Level II

Demitriades et al. ${ }^{33} 2006$

Subgroup $\left(/^{2}=. \%\right)$

Lower levels

Richardson et al. ${ }^{44} 1997$

Demitriades et al. 332006 level III

Roubik et al., ${ }^{42} 2017$ level III + IV

Subgroup $\left(/ /^{2}=54.9 \%\right)$

Other

Norwood et al., ${ }^{48} 2011$ PA

Norwood et al., ${ }^{48} 2011$ BA

Norwood et al. ${ }^{48} 2011 \mathrm{PCl}$

Norwood et al. ${ }^{48} 2011$ BC

Smith et al. ${ }^{10} 2011$ ARDS

Roubik et al. 422017 level I+ II

Schlegel et al., 512018 age $<5$ yr

Subgroup $(/ 2=87.7 \%)$

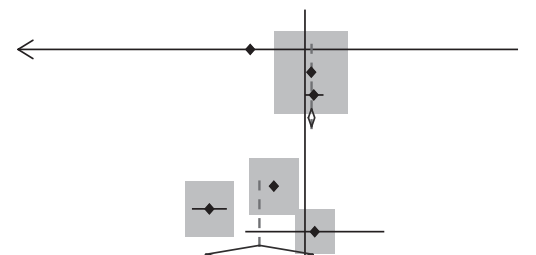

Crude RR $(95 \% \mathrm{Cl})$ Weight, \%*

$0.70(0.12$ to 4.09$)$

$1.04(1.02$ to 1.07$)$

1.06 (1.00 to 1.13$)$

$1.04(1.02$ to 1.07$)$

0.81 (0.79 to 0.83$)$

0.53 (0.47 to 0.60$)$

1.07 (0.67 to 1.69$)$

$0.74(0.52$ to 1.06$)$

0.01

88.08

11.90

100.00

38.91

37.45
23.64

100.00

$0.80(0.78$ to 0.82$)$ $0.60(0.45$ to 0.81$)$

$1.02(0.90$ to 1.16$)$

$1.05(0.66$ to 1.66$)$

$0.60(0.14$ to 2.57$)$

$0.86(0.59$ to 1.25$)$

$0.86(0.59$ to 1.25$)$

$0.97(0.95$ to 0.99$)$
$0.88(0.77$ to 1.00$)$

26.07

26.07
11.08

11.08
21.31

6.23

0.79
8.29
26.22

100.00

0.85 (0.82 to 0.88$) \quad 100.00$

0.85 (0.82 to 0.88$) \quad 100.00$

$1.92(0.64$ to 5.77$) \quad 4.75$

$1.02(0.77$ to 1.36$) \quad 34.95$

$\begin{array}{ll}1.02(0.77 \text { to } 1.36) & 34.95 \\ 0.82(0.76 \text { to } 0.88) & 60.30\end{array}$

0.92 (0.72 to 1.18 ) $\quad 100.00$

$0.62(0.27$ to 1.41$) \quad 7.67$

$0.91(0.48$ to 1.75$) \quad 10.33$

$0.38(0.16$ to 0.88$) \quad 7.29$

$0.41(0.28$ to 0.61$) \quad 16.15$

$0.75(0.65$ to 0.86$) \quad 22.59$

$1.03(1.01$ to 1.06$)$

$0.75(0.43$ to 1.31$)$

$\begin{array}{lc}0.75(0.43 \text { to } 1.31) & 12.11 \\ 0.70(0.53 \text { to } 0.93) & 100.00\end{array}$

0.15

Favours verification

RR

B Population; study

All patients

Grossman et al., ${ }^{49} 2017$ adults

Grossman et al.., ${ }^{49} 2017$ older adults

Subgroup $\left(/^{2}=0.0 \%\right)$

Pediatrics

Osler et al., ${ }^{33} 2001$

Grossman et al., ${ }^{47} 2017$

Subgroup $\left(/^{2}=86.3 \%\right)$

Severe injuries

Grossman et al. 492017 pediatric ISS > 24

Grossman et al., ${ }^{49} 2017$ adults ISS > 24

Grossman et al., 492017 older adults ISS $>24$

Subgroup $\left(/^{2}=0.0 \%\right)$

Level I

Demitriades et al. ${ }^{33} 2006$

Brown et al., ${ }^{16} 2013$

Agrawal et al., ${ }^{7} 2018$

Subgroup $\left(/^{2}=86.4 \%\right)$

Level II

Brown et al ${ }^{16} 2013$

Subgroup $\left(/^{2}=. \%\right)$

Other

$\mathrm{Kim}^{34} 2006$ TBI

Smith et al., ${ }^{10} 2011$ ARDS

Subgroup $\left(/^{2}=x x \times \%\right)$

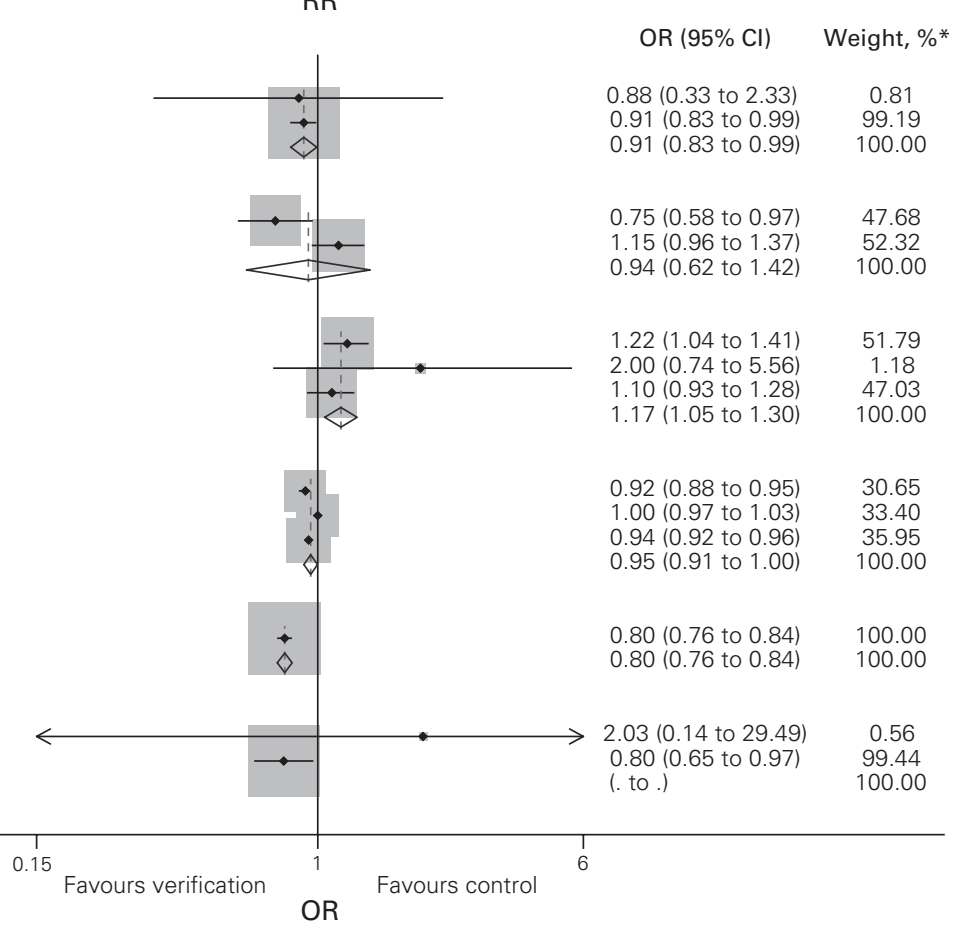

Fig 2. (A) Meta-analysis of crude association between trauma centre verification and in-hospital mortality. (B) Meta-analysis of riskadjusted association between trauma centre verification and in-hospital mortality. Odds ratios (ORs) are presented instead of relative risks (RRs) because it was the effect measure reported by studies with adjusted analyses, and they did not provide enough details to compute adjusted RRs. ARDS = acquired respiratory distress syndrome; $\mathrm{BAl}=$ blunt abdominal injury; $\mathrm{BCl}=$ blunt cardiovascular injury; $\mathrm{Cl}=$ confidence interval; ISS = Injury Severity Score; $\mathrm{PAl}=$ penetrating abdominal injury; $\mathrm{PCl}=$ penetrating cardiovascular injury; $\mathrm{TBI}=$ traumatic brain injury. ${ }^{*}$ From random-effects model. 
A

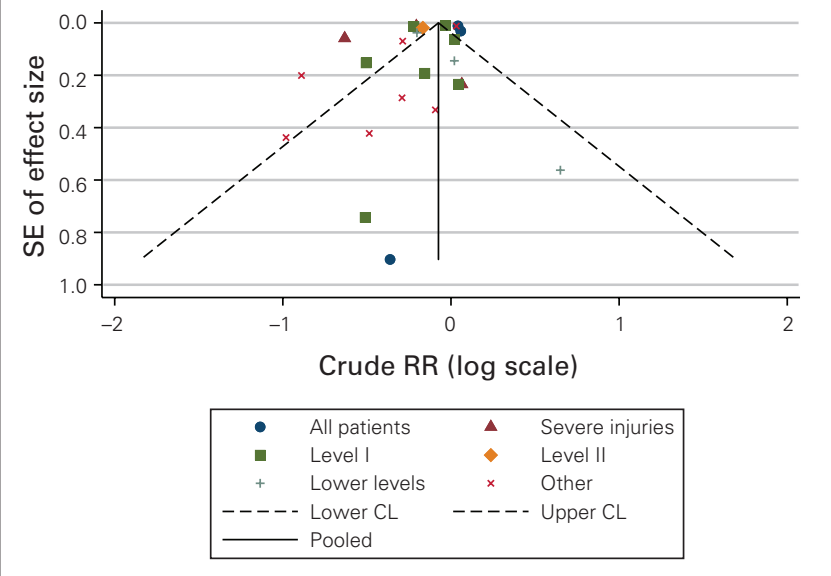

B

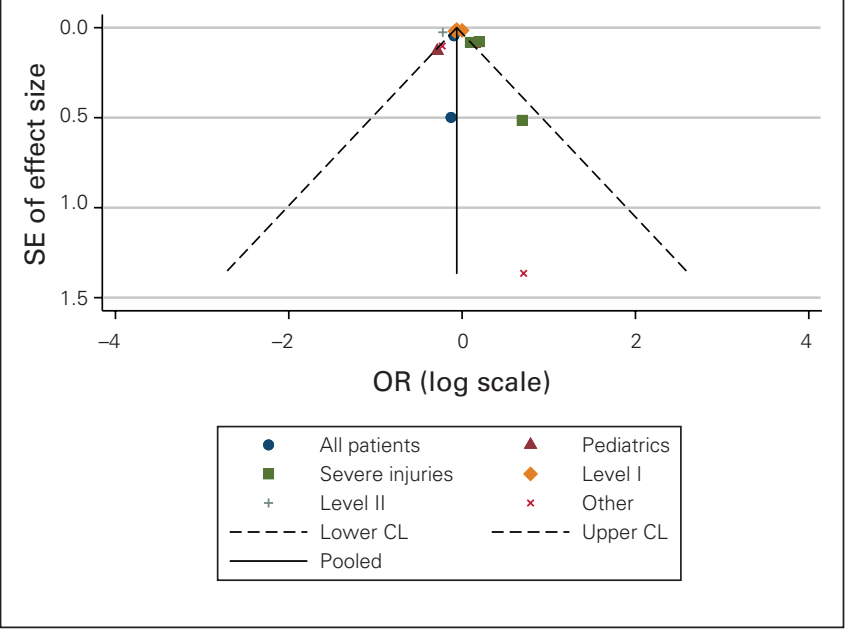

Fig. 3. Funnel plots with pseudo $95 \%$ confidence limits (CLs) of studies showing the crude association between trauma centre verification and in-hospital mortality $(\mathrm{A})$ and of studies showing the adjusted association between trauma centre verification and in-hospital mortality (B). OR = odds ratio; $\mathrm{RR}=$ relative risk; $\mathrm{SE}=$ standard error.

\section{Adverse events}

Of the 7 studies that reported adverse events, ${ }^{7-10,14,45,50}$ 3 adjusted for potential confounders. ${ }^{7,9,14}$ Four studies were cross-sectional, and 3 were pre-post. Two studies focused on injured pediatric patients, 1 study focused on adults, and 1 study focused on both pediatric and adult patients. Investigated outcomes included a wide range of complications such as pneumonia, pulmonary emboli, unplanned intubation, unplanned return to the operating room and unplanned readmission.

We did not conduct a meta-analysis of results for adverse events owing to the diversity of outcomes investigated; rather, we report them narratively. After risk adjustment, Agrawal and colleagues ${ }^{7}$ found lower odds of complications in verified centres than in state-designated centres (OR 0.88, 95\% CI 0.87 to 0.90). Schubert and colleagues ${ }^{9}$ found a positive association between verified centres and unplanned intubation, especially among level I trauma centres (RR 1.53 , 95\% CI 1.11 to 1.65 ), after adjusting for hospital and patient characteristics. They did not observe an association between verification and unplanned return to the operating room. Piontek and colleagues $^{45}$ found low evidence for changes in the incidence of complications (RR 1.27 , 95\% CI 0.86 to 1.89 ) or unplanned 30-day readmission (RR 0.91 , 95\% CI 0.77 to 1.08) after verification of a level II trauma centre. Likewise, Alexander and colleagues ${ }^{50}$ did not find an association between re-verification of a pediatric trauma centre and unplanned 30-day readmission at an already verified adult level I centre. The small number of patients (126) and readmissions (2), however, limit the interpretation of their findings. Conversely, Choi and colleagues ${ }^{8}$ found a decrease in unplanned hospital readmissions (RR $0.36,95 \%$ CI 0.15 to 0.87 ) and hospital-acquired pneumonia (RR 0.41, 95\% CI 0.17 to 0.99$) 2$ years after verification of a level I trauma centre. Grossman and colleagues, ${ }^{14}$ using a representative sample $(n=94)$ of US trauma centres, found that verified centres had a lower incidence of major complications (based on the National Trauma Data Bank definition ${ }^{54}$ ) than nonverified centres. This association was higher among older adults (OR 0.40, 95\% CI 0.27 to 0.60 ) and children with an Injury Severity Score greater than 24 (OR 0.23, 95\% CI 0.12 to 0.47 ). Finally, Smith and colleagues ${ }^{10}$ observed fewer cases of acute respiratory distress syndrome in verified level I trauma centres than in state-designated centres (RR 0.91, 95\% CI 0.84 to 0.99) (Table 2).

\section{Processes of care}

In 4 of the 12 included studies, the authors adjusted for at least 1 potential confounder. ${ }^{34,35,41,52}$ Six studies focused on pediatric patients, ${ }^{39,41,46,49,50,52} 2$ on adults, ${ }^{34,37}$ and 4 on both pediatric and adult patients. ${ }^{34,36,38,44}$ There were 7 crosssectional studies and 5 pre-post designs.

We did not conduct a meta-analysis owing to the diversity of outcomes investigated. In the pediatric population, a reduction in the incidence of splenectomy was found by Murphy and colleagues ${ }^{49}$ (2.7\% among verified trauma centres v. $11 \%$ among nonverified centres) and Alarhayem and colleagues ${ }^{39}$ (6\% among verified trauma centres v. $13 \%$ among nonverified centres) following verification of a pediatric level 1 centre. Alexander and colleagues ${ }^{50}$ observed a decrease in splenic interventions (i.e., splenectomy, splenorrhaphy or embolization) among children with blunt splenic injuries following pediatric verification (RR $0.36,95 \%$ CI 0.13 to 0.99). Ehrlich and colleagues ${ }^{46}$ reported an improvement in pediatric trauma patient evaluation (including radiology) and time to emergency department discharge (<120 min) following verification of a combined adult/pediatric level I trauma centre. Finally, after adjusting for Injury Severity Score, Bogumil and 


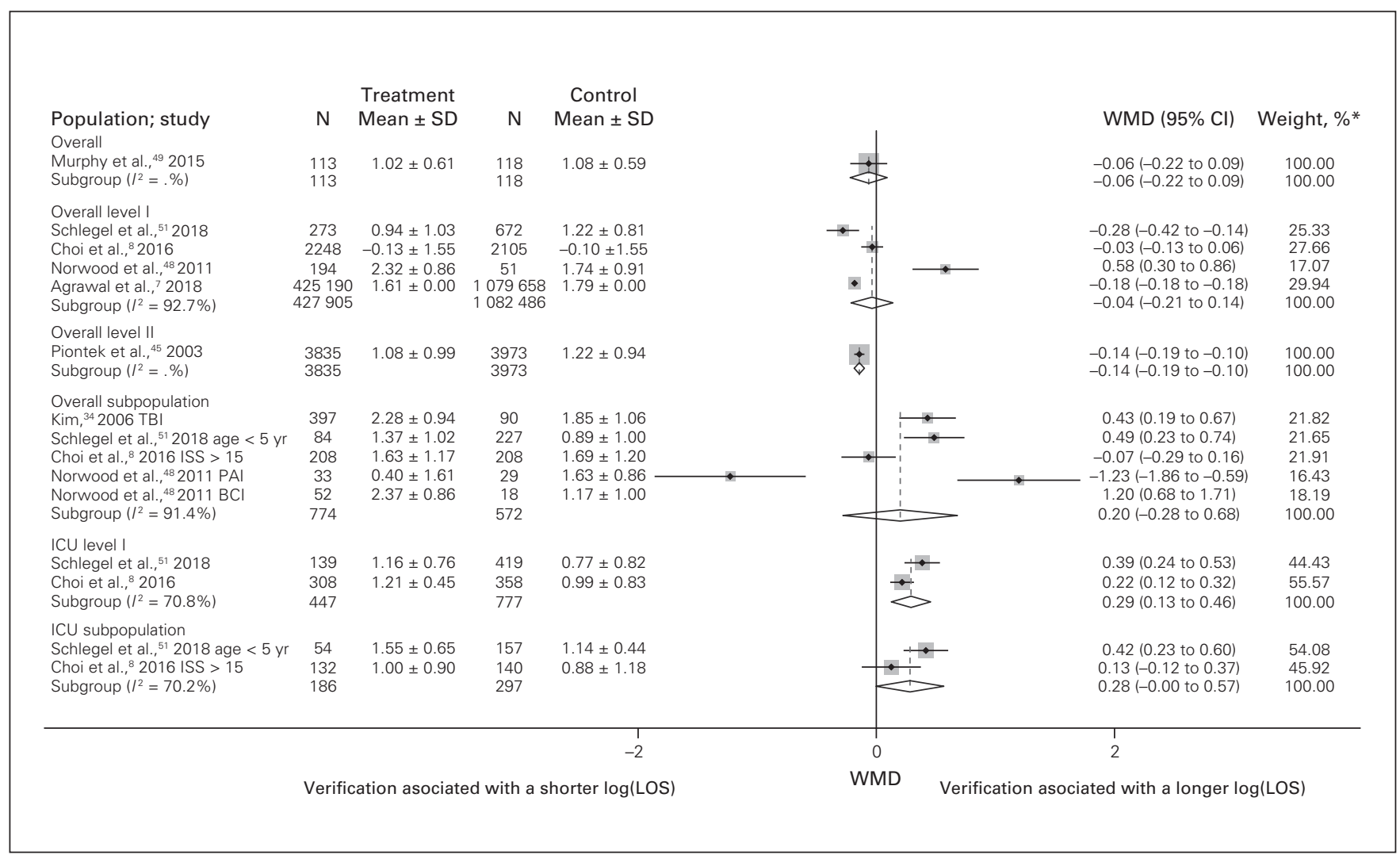

Fig. 4. Meta-analysis of studies reporting an association between trauma centre verification and length of stay (log scale). Overall subpopulation $=$ total length of stay estimate in a subgroup. Exponentiate of weighted mean differences (WMDs) can be interpreted as geometric mean ratio. $\mathrm{BCl}=$ blunt cardiovascular injury; $\mathrm{Cl}=$ confidence interval; ICU = intensive care unit; ISS = Injury Severity Score; PAI = penetrating abdominal injury.

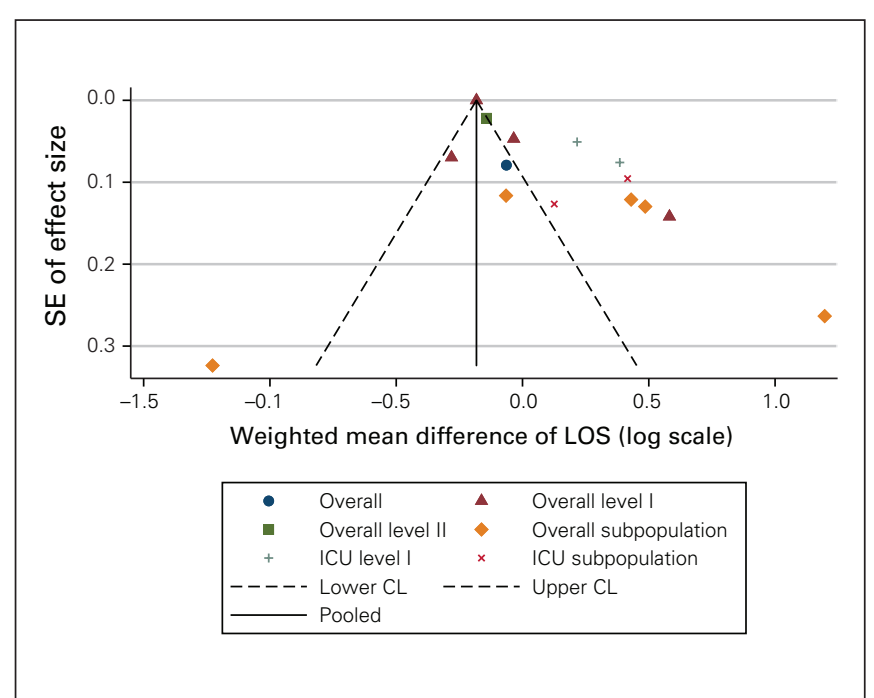

Fig. 5. Funnel plot with pseudo $95 \%$ confidence limits (CLs) of studies reporting the association between trauma centre verification and length of stay (LOS) (log scale). Overall subpopulation $=$ total length of stay estimate in a subgroup. ICU = intensive care unit; $\mathrm{SE}=$ standard error.

colleagues $^{41}$ observed a higher prevalence of nonaccidental trauma in verified pediatric centres than in nonverified centres (prevalence ratio $1.81,95 \%$ CI 1.73 to 1.90 ). This association was higher in level I centres (prevalence ratio $1.89,95 \%$ CI 1.80 to 1.98 ) than level II centres (prevalence ratio $1.62,95 \%$ CI 1.51 to 1.75$)$.

Surveys of all state-designated US trauma centres in 2000 and 2006 showed that verified centres had a higher likelihood of full compliance with published guidelines for the management of severe traumatic brain injury for both 2000 (OR 5.1, 95\% CI 1.1 to 23) and 2006 (OR 1.55, 95\% CI 1.00 to 2.40). ${ }^{32,35}$ Similarly, Theologis and colleagues ${ }^{38}$ reported that verified level I trauma centres had a higher proportion of compliance with cervical spine clearance protocols than nonverified centres $(75 \%$ v. $54 \%)$. $\mathrm{Kim}^{34}$ did not find any association between verification and time to surgery in patients with head injuries. Finally, Richardson and colleagues ${ }^{44}$ found that verification of a level III trauma centre was associated with an increase in the proportion of admissions of transferred patients into a referent level I centre (RR 1.19, 95\% CI 1.03 to 1.36) (Table 2).

\section{Discussion}

In this systematic review and meta-analysis, we found mixed and inconsistent results for the association between trauma centre verification by the American College of Surgeons and all outcomes studied (in-hospital mortality, 
adverse events, resource use and processes of care). Nonetheless, verification was imprecisely associated with decreased mortality and longer LOS. Some evidence pointed to positive associations between verification and some processes of care, including adherence to published guidelines and reductions in the occurrence of complications. These findings, however, should be interpreted with caution given serious methodologic concerns about the quality of the empirical evidence.

First, inference of the obtained estimates is limited by the unclear nature of the control group in each study. For instance, in cross-sectional studies (18/29), it was not possible to distinguish those that failed during the verification process from those that never applied among nonverified centres. In addition, a quarter of multicentre studies $(5 / 18)$ combined centres that had no trauma designation and state-designated centres as nonverified centres. If we placed ourselves in a trial framework, the results obtained from these studies would be neither intention-to-treat nor per-protocol estimates. This issue leads to selection and healthy-user biases, in the sense that high-performing centres may be more willing to seek verification than lowperforming centres. This may affect the validity and generalizability of the observed associations. It is important to note that not all included studies assessed the impact of verification as the primary objective.

Although pre-post studies are less vulnerable to the biases mentioned above, they cannot account for the underlying trend in the measured outcomes before verification, ${ }^{55}$ which can bias estimates in either direction. An interesting alternative to assess verification benefits would be the use of quasiexperimental designs such as differencein-differences and interrupted time-series, which are frequently used to assess the impact of policy and other population-level interventions in health research. These methods can account for unobservable or unmeasured variables that are fixed over time, and for secular trends in outcomes. ${ }^{56,57}$

Second, preparation for verification visits may lead to improvements in measured outcomes and therefore bias estimates of associations. Only 3 studies $^{8,46,51}$ accounted for this, by removing the period just before verification in the analysis or via stratification. Of the 73 articles excluded from our review, 6 were excluded because the authors assessed only the preparation for verification visits. ${ }^{58-63}$

Finally, issues related to analytical methods may have biased the results. For instance, $45 \%$ of studies did not adjust for centre-level or patient-level risk factors. The latter is necessary to account for the changing epidemiologic features of trauma populations (e.g., due to population aging and possible change in referral patterns generally attributed to increased marketability). ${ }^{8}$ In addition, several papers reported ORs as a measure of association, but ORs are known to overestimate RRs, especially when the outcome is common. ${ }^{64}$ Only one-third (6/18) of multicentre studies accounted for this in their analysis, which may have led to type I errors and CIs that were too narrow. ${ }^{31}$ Also, the competing risk of death was not considered when LOS was assessed, and missing data were rarely handled appropriately (Table 3).

Our findings are similar to those of previous systematic reviews assessing verification in other health care fields ${ }^{65-69}$ that showed that many of the studies were heterogeneous and highly vulnerable to confounding, and added little clarity or guidance. They also highlighted major methodologic challenges such as self-selection and lack of robust controls, which limit their inference.

\section{Limitations}

Although the uptake of trauma centre verification is rising worldwide, all included articles were from the US. Pediatric patients were overrepresented. The inclusion of multiple study designs provided a more comprehensive assessment of the relevant literature; however, it introduces substantial heterogeneity, which, in turn, affects the robustness of meta-analysis estimates. Our choice of random-effects meta-analysis was based on the assumption that there might not be a common RR or OR applicable to all trauma populations. ${ }^{70}$ The small number of studies included in our meta-analysis made it difficult to properly summarize estimates and interpret funnel plots. Nonetheless, publication bias seems to be more likely in crude than in adjusted analyses. We also noted that several large studies fell outside the projected lines of the funnel plots, which indicates substantial variability among studies with small SEs. ${ }^{71}$ Since trauma-verification standards have evolved with time, we were unable to stratify our results by time, which may have introduced a bias. ${ }^{3}$ Studies were excluded from meta-analyses because of missing CIs or SEs and the scale of effect measure used, despite our efforts to compute desired statistics when raw data were available. Finally, the quality and strength of the cumulative evidence (as assessed with the GRADE framework) was very low. ${ }^{72}$

\section{Conclusion}

Our review illustrates the inability to extrapolate or infer causality on the effectiveness of trauma centre verification from the published literature owing to significant methodologic challenges, such as the lack of robust controls and the concentration of all the available studies in the US. Considering the prevalence and spread of trauma verification globally, this systematic review and meta-analysis underscores the need for quasiexperimental studies that assess the impact of trauma centre verification on changes in clinical processes of care and outcomes. Such studies may provide solid evidence to guide policy-making and individual hospitals' decisions to seek verification. 
Acknowledgement: The authors acknowledge librarian Andrea Quaiattini for her help in refining the research question, keywords and $\mathrm{MeSH}$ terms for the preliminary search strategy.

Affiliations: From the Department of Epidemiology, Biostatistics, and Occupational Health, McGill University, Montréal, Que. (Batomen, Carabali); the Department of Social and Preventive Medicine, Université Laval, Québec, Que. (Moore) and the Population Health and Optimal Health Practices Research Unit, Trauma - Emergency - Critical Care Medicine, Centre de recherche du CHU de Québec - Université Laval, Québec, Que. (Moore, Tardif); the Department of Surgery, Uniformed Services University of the Health Sciences, Bethesda, Md. (Champion); and the Institute for Health and Social Policy, Department of Epidemiology, Biostatistics, and Occupational Health, McGill University, Montréal, Que. (Nandi).

Competing interests: None declared.

Contributions: B. Batomen, L. Moore, A. Nandi, M. Carabali, P.-A. Tardif and H. Champion designed the study. B. Batomen and M. Carabali acquired the data extraction, which B. Batomen, L. Moore, M. Carabali, H. Champion and A. Nandi analyzed. B. Batomen and M. Carabali drafted the manuscript, which A. Nandi, P.-A. Tardif, L. Moore, M. Carabali, H. Champion and B. Batomen critically revised. All authors gave final approval of the article to be published.

Content licence: This is an Open Access article distributed in accordance with the terms of the Creative Commons Attribution (CC BY-NCND 4.0) licence, which permits use, distribution and reproduction in any medium, provided that the original publication is properly cited, the use is noncommercial (i.e., research or educational use), and no modifications or adaptations are made. See: https://creativecommons.org/ licenses/by-nc-nd/4.0/.

\section{References}

1. Gabbe BJ, Lyons RA, Fitzgerald MC, et al. Reduced population burden of road transport-related major trauma after introduction of an inclusive trauma system. Ann Surg 2015;261:565-72.

2. MacKenzie EJ, Rivara FP, Jurkovich GJ, et al. A national evaluation of the effect of trauma-center care on mortality. N Engl 7 Med 2006; 354:366-78.

3. Committee on Trauma, American College of Surgeons. Resources for optimal care of the injured patient. Chicago: American College of Surgeons; 2014. Available: https://bit.ly/2RWVyFs (accessed 2019 Jan. 21).

4. American College of Surgeons. Resources for optimal care of the injured patient 2014/resources repository. Chicago: American College of Surgeons; 2018. Available: https://bit.ly/1nXDI1p (accessed 2018 Mar. 7).

5. Trauma system accreditation guidelines. Halifax: Trauma Association of Canada; 2011:88.

6. Trauma Distinction information package. Ottawa: Accreditation Canada; 2014. Available: https://accreditation.ca/files/trauma-info -package-en.pdf (accessed 2018 Feb. 16).

7. Agrawal V, Deramo PJ, Lowrance E, et al. ACS verified level I centers have better clinical outcomes than state designated level I trauma centers. Trauma Mon 2018;23:e14435.

8. Choi PM, Hong C, Woods S, et al. Early impact of American College of Surgeons - verification at a level-1 pediatric trauma center. $\mathcal{F}$ Pediatr Surg 2016;51:1026-9.

9. Schubert FD, Gabbe LJ, Bjurlin MA, et al. Differences in trauma mortality between ACS-verified and state-designated trauma centers in the US. Injury 2019;50:186-91.

10. Smith J, Plurad D, Inaba K, et al. Are all level I trauma centers created equal? A comparison of American College of Surgeons and state-verified centers. Am Surg 2011;77:1334-6.

11. Edlich RF. Verified level 1 pediatric trauma centers. Intern Emerg Med 2006;1:300-1.

12. Verification/Consultation Program for Hospitals, Committee on Trauma, American College of Surgeons. Hospital prereview ques- tionnaire (PRQ). Chicago: American College of Surgeons; 2013. Available: http://ow.ly/yoQm30mkGob (accessed 2018 Mar. 6).

13. America Trauma Society. Trauma center levels explained. Available: www.amtrauma.org/?page=TraumaLevels (accessed 2018 Mar. 6).

14. Grossman MD, Yelon JA, Szydiak L. Effect of American College of Surgeons trauma center designation on outcomes: measurable benefit at the extremes of age and injury. 7 Am Coll Surg 2017;225:194-9.

15. Ashley DW, Mullins RF, Dente CJ, et al. What are the costs of trauma center readiness? Defining and standardizing readiness costs for trauma centers statewide. Am Surg 2017;83:979-90.

16. Brown JB, Watson GA, Forsythe RM, et al. American College of Surgeons trauma center verification versus state designation: Are level II centers slipping through the cracks? 7 Trauma Acute Care Surg 2013;75:44-9.

17. Fairbrother G, Gleeson M. EQuIP accreditation: feedback from a Sydney teaching hospital. Aust Health Rev 2000;23:153-62.

18. Rotondo MF, Bard MR, Sagraves SG, et al. What price commitment: What benefit? The cost of a saved life in a developing level I trauma center. 7 Trauma Acute Care Surg 2009;67:915-23.

19. Batomen B, Moore L, Carabali M, et al. Effectiveness of trauma centers verification: protocol for a systematic review. Syst Rev 2019;8:292.

20. Moher D, Liberati A, Tetzlaff J, et al; PRISMA Group. Preferred Reporting Items for Systematic Reviews and Meta-Analyses: the PRISMA Statement. PLoS Med 2009;6:e1000097.

21. Bramer WM, Giustini D, de Jonge GB, et al. De-duplication of database search results for systematic reviews in EndNote. 7 Med Libr Assoc 2016;104:240.

22. Ouzzani M, Hammady H, Fedorowicz Z, et al. Rayyan — a web and mobile app for systematic reviews. Syst Rev 2016;5:210.

23. Zhang J, Yu KF. What's the relative risk? A method of correcting the odds ratio in cohort studies of common outcomes. FAMA 1998; 280:1690-1.

24. Wan X, Wang W, Liu J, et al. Estimating the sample mean and standard deviation from the sample size, median, range and/or interquartile range. BMC Med Res Methodol 2014;14:135.

25. Higgins JPT, White IR, Anzures-Cabrera J. Meta-analysis of skewed data: combining results reported on log-transformed or raw scales. Stat Med 2008;27:6072-92.

26. Sterne JA, Hernán MA, Reeves BC, et al. ROBINS-I: a tool for assessing risk of bias in non-randomised studies of interventions. BM7 2016;355:i4919.

27. Berkman ND, Lohr KN, Ansari M, et al. Grading the strength of a body of evidence when assessing health care interventions for the effective health care program of the Agency for Healthcare Research and Quality: an update. In: Methods guide for effectiveness and comparative effectiveness reviews [Internet]. Rockville (MD): Agency for Healthcare Research and Quality; 2013.

28. Borenstein M, Hedges L, Rothstein H. Meta-analysis: fixed effect vs. random effects. 2007. Available: www.meta-analysis.com (accessed 2019 Sept. 1).

29. Higgins JP, Thompson SG. Quantifying heterogeneity in a metaanalysis. Stat Med 2002;21:1539-58.

30. Sterne JA, Harbord RM. Funnel plots in meta-analysis. Stata 7 2004; 4:127-41.

31. Osler TM, Vane DW, Tepas JJ, et al. Do pediatric trauma centers have better survival rates than adult trauma centers? An examination of the National Pediatric Trauma Registry. F Trauma 2001;50:96-101.

32. Hesdorffer DC, Ghajar J, Iacono L. Predictors of compliance with the evidence-based guidelines for traumatic brain injury care: a survey of United States trauma centers. F Trauma 2002;52:1202-9.

33. Demetriades D, Martin M, Salim A, et al. Relationship between American College of Surgeons trauma center designation and mortality in patients with severe trauma (Injury Severity Score $>15$ ). 7 Am Coll Surg 2006;202:212-5.

34. Kim Y. Time to surgery and outcomes in patients with head injury [dissertation]. Baltimore: University of Maryland; 2006. 
35. Hesdorffer DC, Ghajar J. Marked improvement in adherence to traumatic brain injury guidelines in United States trauma centers. 7 Trauma 2007;63:841-8.

36. Horton JD, Dezee KJ, Wagner M. Use of rFVIIa in the trauma setting - practice patterns in United States trauma centers. Am Surg 2008;74:413-7.

37. Notrica DM, Weiss J, Garcia-Filion P, et al. Pediatric trauma centers: correlation of ACS-verified trauma centers with CDC statewide pediatric mortality rates. 7 Trauma Acute Care Surg 2012;73:566-72.

38. Theologis AA, Dionisio R, Manley G, et al. Current clinical protocols for cervical spine clearance in level I trauma centers in the United States. 7 Am Coll Surg 2012;215:S63.

39. Alarhayem AQ, Liao LF, Stewart RM, et al. Management of pediatric splenic injuries: a nationwide analysis. FACS 2015;221:e143-4.

40. Russell J, Tomanec A, Leeson B, et al. Bedside ultrasound has become standard of care in the evaluation of pediatric trauma patients in the United States. Acad Emerg Med 2015;22:S273.

41. Bogumil DDA, Demeter NE, Imagawa KK, et al. Prevalence of nonaccidental trauma among children at American College of Surgeons-verified pediatric trauma centers. 7 Trauma Acute Care Surg 2017;83:862-6.

42. Roubik D, Cook AD, Ward JG, et al. Then we all fall down: fall mortality by trauma center level. 7 Surg Res 2017;217:36.

43. Jenkins PC, Painter S, Bell TM, et al. The conference effect: national surgery meetings are associated with increased mortality at trauma centers without American College of Surgeons verification. PLoS One 2019;14:e214020.

44. Richardson JD, Cross T, Lee D, et al. Impact of level III verification on trauma admissions and transfer: comparisons of two rural hospitals. F Trauma 1997;42:498-502.

45. Piontek FA, Coscia R, Marselle CS, et al. Impact of American College of Surgeons verification on trauma outcomes. 7 Trauma 2003; 54:1046-7.

46. Ehrlich PF, McClellan WT, Wesson DE. Monitoring performance: longterm impact of trauma verification and review. 7 Am Coll Surg 2005;200:166-72.

47. Maggio PM, Brundage SI, Hernandez-Boussard T, et al. Commitment to COT verification improves patient outcomes and financial performance. 7 Trauma 2009;67:190-4.

48. Norwood S, Cook AD, Berne JD. Level I verification is associated with a decreased mortality rate after major torso vascular injuries. $\mathrm{Am}$ Surg 2011;77:32-7.

49. Murphy EEK, Murphy SG, Cipolle MD, et al. The pediatric trauma center and the inclusive trauma system: impact on splenectomy rates. 7 Trauma Acute Care Surg 2015;78:930-3.

50. Alexander M, Zaghal A, Wetjen K, et al. Pediatric trauma center verification improves quality of care and reduces resource utilization in blunt splenic injury. 7 Pediatr Surg 2019;54:155-9.

51. Schlegel C, Greeno A, Chen H, et al. Evolution of a level I pediatric trauma center: changes in injury mechanisms and improved outcomes. Surgery 2018;163:1173-7.

52. Abd El-Shafy I, Zapke J, Sargeant D, et al. Decreased pediatric trauma length of stay and improved disposition with implementation of Lewin's change model. 7 Trauma Nurs 2019;26:84-8.

53. Notrica DM, Sayrs LW, Krishna N. The effect of verified pediatric trauma centers, state laws, and crash characteristics on time trends in adolescent motor vehicle fatalities, 1999-2015. 7 Trauma Acute Care Surg 2018;85:944-52.
54. American College of Surgeons Committee on Trauma Leadership National Trauma Data Bank 2016: annual report. Available: https://www.facs.org/-/media/files/quality-programs/trauma/ntdb/ ntdb-annual-report-2016.ashx (accessed 2020 Dec. 23).

55. Donnelly NJ. The use of interrupted time series analysis to evaluate the impact of pharmaceutical benefits scheme policies on drug utilisation in Australia [dissertation]. Sydney (Australia): University of New South Wales; 2005.

56. Zhou H, Taber C, Arcona S, et al. Difference-in-differences method in comparative effectiveness research: utility with unbalanced groups. Appl Health Econ Health Policy 2016;14:419-29.

57. Bernal JL, Cummins S, Gasparrini A. Interrupted time series regression for the evaluation of public health interventions: a tutorial. Int $\mathcal{F}$ Epidemiol 2017;46:348-55.

58. Biffl WL, Harrington DT, Majercik SD, et al. The evolution of trauma care at a level I trauma center. 7 Am Coll Surg 2005;200:922-9.

59. DiRusso S, Holly C, Kamath R, et al. Preparation and achievement of American College of Surgeons level I trauma verification raises hospital performance and improves patient outcome. 7 Trauma 2001; 51:294-9.

60. Ehrlich PF, Rockwell S, Kincaid S, et al. American College of Surgeons, Committee on Trauma verification review: Does it really make a difference? $\mathcal{F}$ Trauma 2002;53:811-6.

61. Nikolis NM, Macwan S, Stein A, et al. Establishing a massive transfusion protocol (MTP): a collaborative effort. Transfusion 2015;55: 213A-4A.

62. Simons R, Kasic S, Kirkpatrick A, et al. Relative importance of designation and accreditation of trauma centers during evolution of a regional trauma system. $\mathcal{F}$ Trauma 2002;52:827-33.

63. Testerman GM, Harris RM, West M, et al. Full-time orthopedic traumatologists enhance rural trauma center pelvic fracture outcomes and financials. Am Surg 2011;77:716-9.

64. Kalilani L, Atashili J. Measuring additive interaction using odds ratios. Epidemiol Perspect Innov 2006;3:5.

65. Greenfield D, Braithwaite J. Health sector accreditation research: a systematic review. Int 7 Qual Health Care 2008;20:172-83.

66. Greenfield D, Pawsey M, Hinchcliff R, et al. The standard of healthcare accreditation standards: a review of empirical research underpinning their development and impact. BMC Health Serv Res 2012;12:329.

67. Brubakk K, Vist GE, Bukholm G, et al. A systematic review of hospital accreditation: the challenges of measuring complex intervention effects. BMC Health Serv Res 2015;15:280.

68. Alkhenizan A, Shaw C. Impact of accreditation on the quality of healthcare services: a systematic review of the literature. Ann Saudi Med 2011;31:407.

69. Hinchcliff R, Greenfield D, Moldovan M, et al. Narrative synthesis of health service accreditation literature. BM7 Qual Saf 2012;21:979-91.

70. Chaimani A, Mavridis D, Salanti G. A hands-on practical tutorial on performing meta-analysis with Stata. Evid Based Ment Health 2014;17: 111-6.

71. Lin HH, Ezzati M, Murray M. Tobacco smoke, indoor air pollution and tuberculosis: a systematic review and meta-analysis. PLoS Med 2007;4:e20.

72. Guyatt G, Oxman AD, Akl EA, et al. GRADE guidelines: 1. Introduction - GRADE evidence profiles and summary of findings tables. 7 Clin Epidemiol 2011;64:383-94. 